\title{
Combined Low Laser Light Phototherapy and Growth Factor Hair Formulation Infiltration Therapy in Androgenetic Alopecia (AGA Combo Treatment)
}

\author{
Luigi Laino* \\ Latuapelle Dermatologic Center, Rome, Italy \\ *Corresponding author: Luigi Laino, Latuapelle Dermatologic Center, Rome, Italy, E-mail: info@latuapelle.it
}

Received: 18 Apr, 2019 | Accepted: 03 May, 2019 | Published: 10 May, 2019

Citation: Laino L (2019) Combined Low Laser Light Phototherapy and Growth Factor Hair Formulation Infiltration Therapy in Androgenetic Alopecia (AGA Combo Treatment). J Clin Cosmet Dermatol 3(2): dx.doi.org/10.16966/2576-2826.140

Copyright: (C) 2019 Laino L. This is an open-access article distributed under the terms of the Creative Commons Attribution License, which permits unrestricted use, distribution, and reproduction in any medium, provided the original author and source are credited.

\begin{abstract}
Alopecia is a hair follicular disorder affecting more than half of the world's population. Androgenetic Alopecia (AGA) is the most common subtype of alopecia that affects over $50 \%$ of men aged over 40 and $75 \%$ of women aged over 65 .

Low-level Light therapy (Laser or LED) is a new technique for stimulating hair growth in men and women that has recently been approved by the US food and drug administration. It is assumed to stimulate anagen phase re-entry in telogen hair follicles, prolong the duration of anagen phase, increase rates of proliferation in active anagen hair follicles and prevent premature catagen development. Industrial Growth Factors Formulation compounds, in locoregional injection therapy, have emerged as a promising regenerative therapy for androgenetic alopecia (AGA). To date, injections of both PRP and Industrial Growth Factors Formulation compounds have been administered to hair loss patients and positive results have been observed. Given the lack of a randomized controlled clinical trial with an appropriate design on the subject, the present study was conducted to carry out a comparative assessment of the effectiveness of low-level led-light therapy added with intradermal injections of a recombinant, bioengineered hair formulation, containing Growth Factors Hair Formulation (we decided to abbreviate in GFHF) in a sterile solution, into the scalp skin, for enhancement of hair regrowth, and evaluate its efficacy, compared to only GFHF therapy (carried out in the same way), for the treatment of alopecia.
\end{abstract}

\section{Introduction}

Alopecia is a hair follicular disorder affecting more than half of the world's population. Androgenetic alopecia (AGA) is the most common subtype of alopecia that affects over $50 \%$ of men aged over 40 and $75 \%$ of women aged over 65 . In genetically-predisposed individuals, androgenetic alopecia is often caused by androgens such as dihydrotestosterone. The most used techniques for the treatment of androgenetic alopecia include the use of topical minoxidil, the administration of Finasteride tablets and hair transplantation. Studies suggest that traditional therapies are only slightly effective in the treatment of this condition. Patients who respond poorly to these common therapies and those who experience side-effects are always seeking more modern and suitable techniques to treat their condition.

Low LED Light Phototherapy as well as Low Laser Light Therapy, is a new technique for stimulating hair growth in men and women that has recently been approved by the US food and drug administration [1]. It is assumed to stimulate anagen phase re-entry in telogen hair follicles, prolong the duration of anagen phase, increase rates of proliferation in active anagen hair follicles and prevent premature catagen development. Most studies conducted on the effectiveness of low-level light therapy in the treatment of alopecia have had epidemiological problems such as lacking scientific rigor, the presence of confounding factors and inappropriate statistical analysis, which render their results unreliable [2].
LLLT, phototherapy or photobiomodulation refers to the use of photons at a non-thermal irradiance to alter biological activity. LLLT uses either coherent light sources (lasers) or non-coherent light sources consisting of filtered lamps or Light-emitting Diodes (LED) or, on occasion, a combination of both. The main medical applications of LLLT are reducing pain and inflammation, augmenting tissue repair and promoting regeneration of different tissues and nerves, and preventing tissue damage in situations where it is likely to occur $[3,4]$. Therefore is possible to define LLLT both coherent Laser source and incoherent LED source.

Industrial Growth Factors Hair Formulation compounds, in locoregional injection therapy, have emerged as a promising regenerative therapy for androgenetic alopecia (AGA) [5]. To date, injections of both PRP and Industrial GFHF have been administered to hair loss patients, and positive results have been observed. However, little to no work has yet to be seen where GFHF treatments are combined with LLLT LED therapy.

Research has shown the efficacy of growth factors formulation in hair regrowth. These industrial preparations of follicular growth factor, allow to have available, a constantly stable and sterile therapy, which therefore maintains constant therapeutic standards and reduces the bias based on the difficulty of preparation of a standardized solution, both in concentration and in the active principles, typical of 
non-industrial autologous substances, such as PRP. Given the lack of a randomized controlled clinical trial with an appropriate design on the subject, the present study was conducted to carry out a comparative assessment of the effectiveness of low-level led-light phototherapy added with intradermal injections of a recombinant, bioengineered hair formulation, containing GFHF in a sterile solution, into the scalp skin, for enhancement of hair regrowth, and evaluate its efficacy, compared to only GFHF therapy (carried out in the same way), for the treatment of alopecia.

\section{Inclusion Criteria}

The inclusion criteria consisted of at least 6 months history of hair loss or thinning without response or with poor response to other commonly used treatments, having no other hair loss disorders, a Fitzpatrick skin type of 1-4, a Norwood-Hamilton grade of 3-6 for men and a Ludwig grade of 2-3 for women and having no history of finasteride and anti-androgen drugs' administration, such as cyproterone, spironolactone, ketoconazole and flutamide, topical estrogen, progesterone, tamoxifen, anabolic steroids, drugs potentially causing hypertrichosis, such as cyclosporine, diazoxide, phenytoin, oral glucocorticoids, and phenothiazines. The average age of the patients was 32 years, with $60 \%$ of male patients and $40 \%$ of women.

\section{Materials and Methods}

In this study, androgenetic alopecia was diagnosed clinically and based on the Ludwig and the Hamilton-Norwood scales and VideoTrichoscopy patterns [6].

The case group (16 patients) received on their bald areas (Figure 1), for six months 4 sessions per month of micro-infiltrative therapy with a Restructuring Hair Booster-natural Growth Factor Formulation (GFF) industrial product containing:

- Uncross-linked hyaluronic acid

- Fibroblast growth factors+polypeptide+NaDNA (N-acetyl-2,3dehydro-2-deoxyneuraminic acid)

- Amino acids: arginine, alanine, aspartic acid, glutamic acid, glycine, histidine, hydroxyproline, isoleucine, lysine, methionine, phenylalanine, proline, serine, threonine, tyrosine, leucine, valine;

- Trace elements: Si, Ca, Fe, K, Mg, Mn, Na, P, SE;

- Vitamins: B12, B9, B3, B5, A, E, B8;

- Terpenes: ginkgolides A,B,C,M, Bilobalide;

- Fatty acids: linoleic acid, oleic acid;

- Flavonoids: quercetin, Kaempferol;

- Antioxidants: Vitamin E, chlorogenic acid, gallic acid, quercetin, kaempferol.

Followed by a 20 -minute sessions ( 4 sessions per month for 24 weeks) of LLLT therapy Using LED Photodynamic Therapy (Figure 1).

The LLLT device and is:

- Kernel - KN-7000A

- Technical data during a single treatment:

- Session Time: 20 minutes

- Range of emission: $633 \mathrm{~nm} \pm 10 \mathrm{~nm}$

- Power of emission: $80 \mathrm{~mW} / \mathrm{cm}^{2} \pm 20 \%$

- Type of emission: continue emission
The experimental group (12 patients) received only industrial FGHF in the same manner.

\section{Results}

The results obtained showed a clinical (Figure 2), and trichoscopic (Figure 3), significantly higher percentage of recovery from androgenetic alopecia in the case group, compared to the control group, and the cases were generally more satisfied with their treatment compared to the controls. the case group revealed a significant increase in this percentage at 6,9 and 12 months after the intervention no side effects in terms of pain, itching, tingling, discomfort, edema, excoriations, necrosis, fissures, erosions, eczema, were detected in all patients, both in the very next days and in the short to medium term.

\section{Statistical Analysis}

The data were analyzed using SPSS. The quantitative data were expressed as mean \pm standard deviation, and the qualitative data as frequency and percentage. The data obtained were compared using the paired t-test, the repeated measures analysis of variance and the independent sample t-test. Given the significance level of 95\%, all results with $\mathrm{p}$-values lower than 0.05 were considered statistically significant.
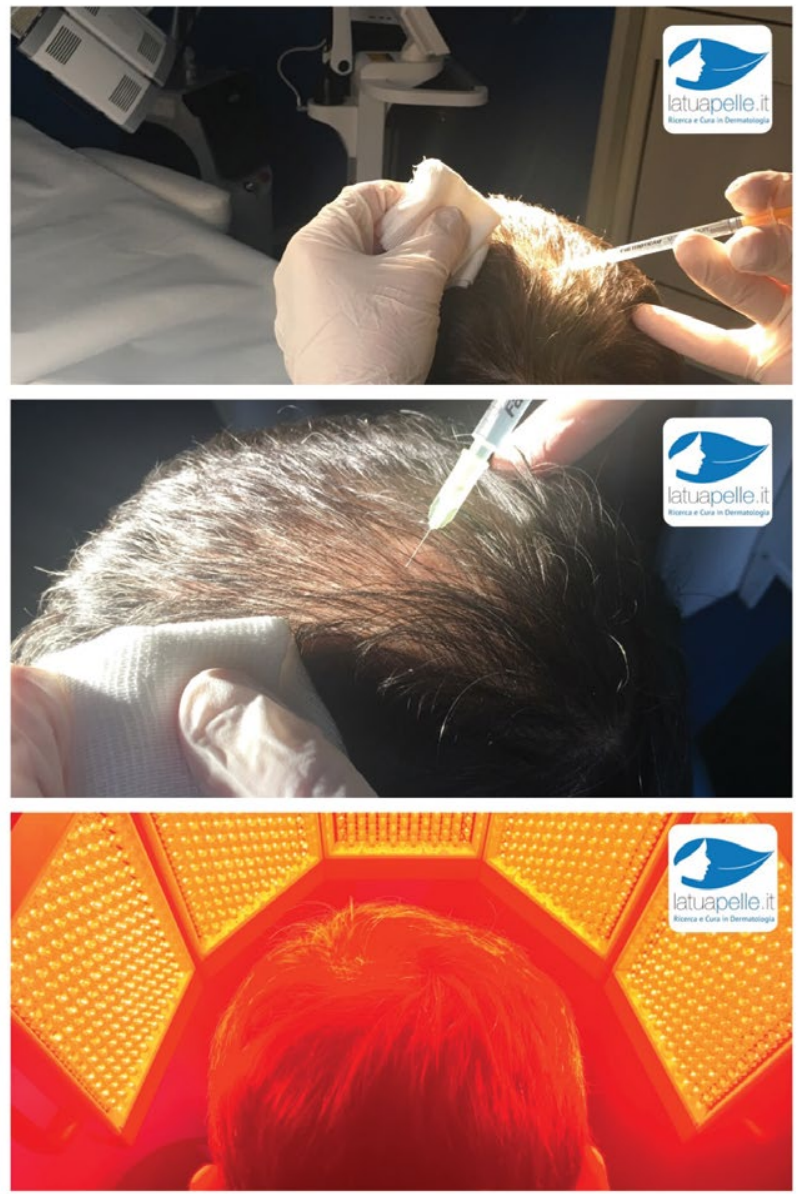

Figure 1: Method: in the first and second box at the top it is possible to appreciate the sub-dermal infiltration method, while in the lower box, the mode of exposure to LLLT. 

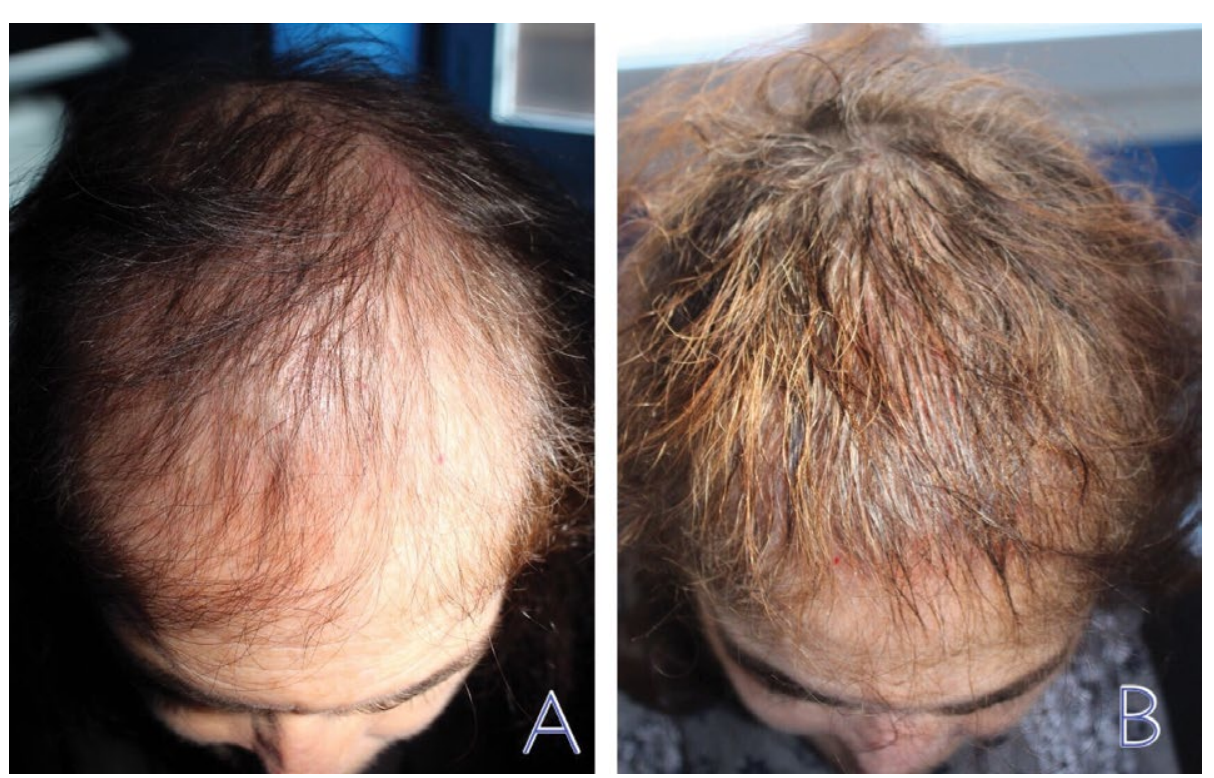

Figure 2: Clinical improvement: before therapy (A) and, after therapy (B): note the increase in the hair regrowth and in hair density, on the area involved by the treatment.

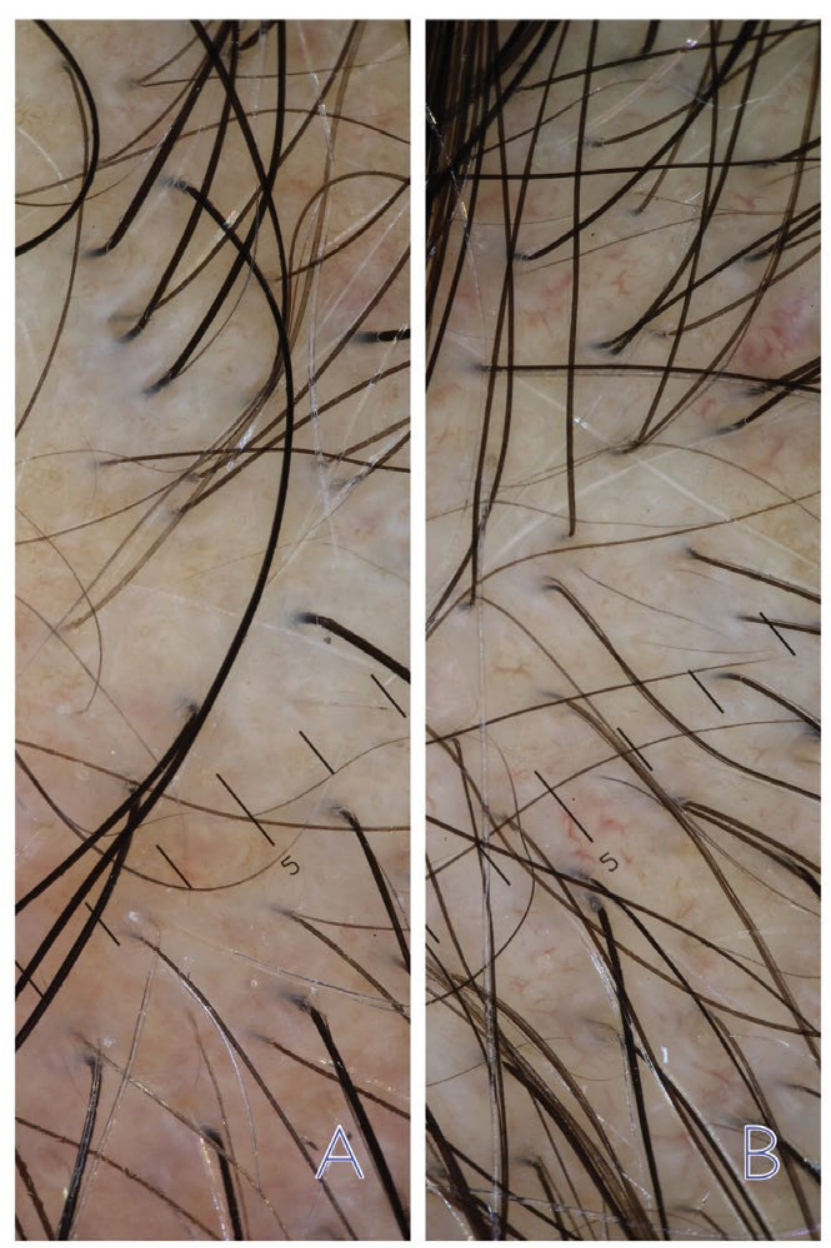

Figure 2: Thrichoscopic improvement: before therapy $(A)$ and, after therapy $(B)$ : notice the increase in local density and average hair thickness in the analyzed area.

\section{Statistical analysis results}

No significant differences were observed between the control and case groups in terms of age $(30.1 \pm 5.7$ vs $30.4 \pm 6.5$ years; $\mathrm{P}=0.9)$ and gender distribution or the male to female ratio $(6.17$ vs $9.13 ; \mathrm{P}=0.29)$.

The percentage of recovery from androgenetic alopecia was similar in both groups 3 months after the intervention; however, compared to the controls, the case group revealed a significant increase in this percentage at 6,9 and 12 months after the intervention $(\mathrm{p}<0.001)$ (Table 1).

\section{Discussion}

For many years, topical and systemic therapies for androgenetic alopecia have remained at stake, hence the need to find alternative routes [7]. The undesirable effects of the same therapies have been for a long time, the subject of long debates and to date, we can say that the tolerability and safety profile is still acceptable, but not devoid of undesirable effects for a small percentage of patients.

Recently, the numerous studies carried out on what are now called "follicular regenerative therapies" have pushed public attention to the choice of the best medical approach to obtain the best results. Certainly, the tolerability and safety profile of micro-infiltrative therapies with regenerative substances and of Low Laser Led Therapy, led the authors to consider their associated use, even in a single session.

The hypothesized mechanisms of action of LLLT are increased Adenosine Triphosphate (ATP) production, modulation of Reactive Oxygen Species (ROS), and induction of transcription factors. The proposed cellular chromosphere responsible for the effect of visible light is Cytochrome C Oxidase (COX) with absorption peaks in the near infrared and mitochondria the likely site for the initial effects. It is believed that LLLT displaces nitric oxide from COX allowing an influx of oxygen to bond to COX and progress forward in the respiratory process to ATP production and ROS signaling. These effects in turn lead to increased cellular proliferation, modulation in levels of cytokines, growth factors and inflammatory mediators, and increased tissue oxygenation [8]. 
Table 1: Recovery means change trough clinical and trichoscopic evaluation in density/thickness defined by alopecia score from the beginning.

\begin{tabular}{|l|c|c|c|c|}
\hline Occasion & Recovery & $\begin{array}{c}\text { Case Group } \\
\mathbf{n}(\%)\end{array}$ & $\begin{array}{c}\text { Control Group } \\
\mathbf{n}(\%)\end{array}$ & $\mathbf{p}$ \\
\hline 3 Months & Grade 1 & $16(100)$ & $12(100)$ & $1<0.001$ \\
\hline \multirow{2}{*}{6 Months } & Grade 1 & 0 & $8(66.66)$ & - \\
\cline { 2 - 5 } & Grade 2 & $16(100)$ & $4(33.34)$ & - \\
\hline \multirow{2}{*}{ 9 Months } & Grade 2 & $1(6.25)$ & $12(100)$ & - \\
\cline { 2 - 5 } & Grade 3 & $15(93.75)$ & - & - \\
\hline \multirow{3}{*}{ 12 Months } & Grade 2 & 0 & $10(83,33)$ & - \\
\cline { 2 - 5 } & Grade 3 & $12(75)$ & $2(16.67)$ & - \\
\cline { 2 - 5 } & Grade 4 & $4(25)$ & - & - \\
\hline
\end{tabular}

( 0 =Poor, 1=Fair, 2=Good, 3=Very good, 4=Excellent)

The hypothesized mechanisms of action of Intradermal Growth Factor Hair Formulation in the promotion of hair growth, as well as other natural compounds as PRP, is still not completely defined: these product, as hypothesis, can induce the proliferation of Dermal Papilla (DP) cells by activating Extracellular Signal-related Kinase (ERK) and Protein Kinase B (Akt, an anti-apoptotic signaling molecule) signaling, EGF and PDGF in PRP up regulate the ERK pathway, leading to the increased transcription of genes involved in cellular proliferation and differentiation [5].

In this study, the authors preferred to choose an labeled industrialtype follicular regenerative product, which always maintained the same preparation and concentration of elements, instead of the autologous PRP, in order to reduce the bias between the possible differences in concentration of the substances and molecules active in the PRP, which notoriously occurs, in the absence of a defined and shared standardization of the same and also due to the real difficulty of preparing a PRP with standard concentrations and stability [9].

\section{Conclusion}

The use of both FGHF plus LLLT to treat AGA could constitute a new frontier for the treatment of Androgenetic Alopecia, given their practicality of use and their high tolerability and safety; this combined therapy is promising based on the results. Safety issues, side effects, and downtime seem to be minimal or absent. However, the optimal treatment protocol for androgenetic alopecia remains to be established, but these regenerative therapies seem to bring a new positive boost in the determination of a unique protocol of care shared by the entire scientific community and within specific guidelines and behavior. Despite the positive results of this study, the limited nature of its sample makes it necessary further investigation.

\section{References}

1. Avci P, Gupta GK, Clark J, Wikonkal N, Hamblin MR (2014) Low-level laser (light) therapy (LLLT) for treatment of hair loss. Lasers Surg Med 46: 144-151.

2. Kim H, Choi JW, Kim JY, Shin JW, Lee SJ, et al. (2013) Low-level light therapy for androgenetic alopecia: A 24-week, randomized, doubleblind, sham device-controlled multicenter trial. Dermatol Surg 39: 1177-1183.

3. Chung H, Dai T, Sharma SK, Huang YY, Carroll JD, et al. (2012) The nuts and bolts of low-level laser (light) therapy. Ann Biomed Eng 40: 516-533.

4. Gupta A, Avci P, Sadasivam M, Chandran R, Parizotto N, et al. (2012) Shining light on nanotechnology to help repair and regeneration. Biotechnol Adv 31: 607-631.

5. Kapoor R, Shome D (2018) Intradermal injections of a hair growth factor formulation for enhancement of human hair regrowth-safety and efficacy evaluation in a first-in-man pilot clinical study. J Cosmet Laser Ther 20: 369-379.

6. Pirmez R, Tosti A (2018) Trichoscopy Tips. Dermatol Clin 36: 413-420.

7. Santos LDN, Shapiro J (2019) What's New in Hair Loss. Dermatol Clin 37: 137-141.

8. Lanzafame RJ, Blanche RR, Bodian AB, Chiacchierini RP, FernandezObregon A, et al. (2013) The growth of human scalp hair mediated by visible red light laser and LED sources in males. Lasers Surg Med 45: 487-495.

9. Frautschi RS, Hashem AM, Halasa B, Cakmakoglu C, Zins JE (2017) Current Evidence for Clinical Efficacy of Platelet Rich Plasma in Aesthetic Surgery: A Systematic Review. Aesthet Surg J 37: 353-362. 